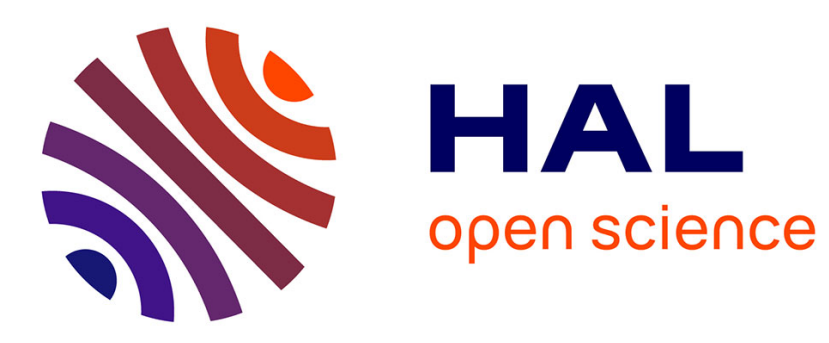

\title{
La coproduction locale de la sécurité en France : un partenariat interinstitutionnel déficient
}

Virginie Gautron

\section{To cite this version:}

Virginie Gautron. La coproduction locale de la sécurité en France : un partenariat interinstitutionnel déficient. Champ Pénal, 2010, Vol. VII, http://champpenal.revues.org/7719. 10.4000/champpenal.7719 . halshs-01075664

\section{HAL Id: halshs-01075664 \\ https://shs.hal.science/halshs-01075664}

Submitted on 19 Oct 2014

HAL is a multi-disciplinary open access archive for the deposit and dissemination of scientific research documents, whether they are published or not. The documents may come from teaching and research institutions in France or abroad, or from public or private research centers.
L'archive ouverte pluridisciplinaire HAL, est destinée au dépôt et à la diffusion de documents scientifiques de niveau recherche, publiés ou non, émanant des établissements d'enseignement et de recherche français ou étrangers, des laboratoires publics ou privés. 


\title{
La coproduction locale de la sécurité en France : un partenariat interinstitutionnel déficient
}

\author{
Virginie Gautron
}

\begin{abstract}
Résumé
Les politiques de lutte contre la délinquance mobilisent de nombreux professionnels au niveau local : préfets, policiers, magistrats, maires, associations, etc. Pour structurer les interdépendances complexes qui se sont nouées entre institutions, une nouvelle doctrine d'action publique s'est progressivement imposée : la «coproduction » de la sécurité. Depuis le début des années 1980, les pouvoirs publics ont institutionnalisé de multiples dispositifs partenariaux pour inciter les acteurs à dialoguer sur leurs missions respectives, définir des orientations communes, mutualiser leurs savoir-faire, leurs ressources et coordonner leurs actions. Si de nombreux sites offrent l'exemple de collaborations réussies, d'échanges interinstitutionnels réguliers et constructifs, les dispositifs de concertation n'ont le plus souvent modifié qu'à la marge les modes relationnels des différents acteurs. Les divergences de représentations, de valeurs, de normes d'action et d'intérêts entravent l'émergence de rapports de complémentarité sur la scène locale. Si les professionnels dialoguent, s'associent ou se sollicitent ponctuellement, notamment dans des situations d'urgence, leurs échanges demeurent bien souvent sporadiques, s'essoufflent au fil du temps et/ou visent simplement la défense de leurs intérêts respectifs et de leurs pratiques habituelles.
\end{abstract}

\begin{abstract}
Public policies of crime prevention and control involve many professionals at the local level : police chiefs and officers, judges, local government and charitable organizations, etc. In order to manage more effectively the complex inter-relationships, which developed between these organizations, a doctrine of public action known as "joint security responsibility" gradually emerged. Since the early 1980s, public authorities have set up many partnerships to encourage the different parties to share their experiences, define common objectives, exchange knowhow and resources, and coordinate their actions. Although many attempts at cooperation have been successful, with regular positive exchanges between institutions, most of the time the relationships between the parties have not evolved. Differences of opinions, values, and methods of action hinder collaboration at the local level. Professionals may talk, get together or appeal to one another as the need arises, in particular in emergency situations, however their exchanges are often sporadic, get weaker as time goes by and/or simply aim at defending their own interests and usual practices.
\end{abstract}


Les politiques de lutte contre la délinquance mobilisent un vaste panel de professionnels au niveau local : préfets, policiers et gendarmes, magistrats, enseignants, maires, travailleurs sociaux, associations, etc. Loin du paradigme weberien qui fait de la violence légitime un monopole d'État, l'heure est à la «coproduction de la sécurité ». Ensemble, les acteurs doivent évaluer les problèmes d'insécurité, définir des orientations communes, mutualiser leurs savoir-faire, leurs ressources et coordonner leurs actions. Cette synergie institutionnelle est recherchée au plus près du terrain, essentiellement au niveau municipal et intercommunal, de façon à produire un meilleur ajustement aux besoins, une gestion adaptée aux réalités et contraintes locales, ainsi qu'un suivi plus fin des programmes. Pour aménager des espaces de dialogue et institutionnaliser l'action collective, les pouvoirs publics ont créé de multiples enceintes de concertation: Conseils locaux de sécurité et de prévention de la délinquance (CLSPD), Groupes locaux de traitement de la délinquance (GLTD), Conseils départementaux de prévention (CDP), etc. Les acteurs tentent d'y construire une stratégie collective, un projet éventuellement formalisé par la signature de contrats locaux de sécurité (CLS). Ces dispositifs partenariaux engagent une méthode d'action coopérative fondée sur un engagement libre, mutuel et contractuel d'acteurs différents mais égaux, qui constituent un acteur collectif dans la perspective d'un changement des modalités de l'action - faire autrement ou faire mieuxsur un objet commun - de par sa complexité et/ou le fait qu'il transcende le cadre d'action de chacun des acteurs -, et élaborent à cette fin un cadre d'action adapté au projet qui les rassemble, pour agir ensemble à partir de ce cadre (Dhume, 2001, 106).

La doctrine de la coproduction véhicule des représentations, des valeurs et des normes d'action positives et séduisantes. Les registres discursifs mobilisés pour la défendre affichent les vertus du consensus, de la cohérence, de la cohésion et de la complémentarité. Sur la base d'un fonctionnement réticulaire, des acteurs devenus partenaires échangeraient sur leurs pratiques, communiqueraient leurs informations respectives, coordonneraient leurs interventions et s'attacheraient à évaluer les résultats de l'action ainsi coproduite. Les recherches menées sur le sujet invitent à plus de prudence. Les dispositifs de coproduction qui se sont succédé depuis le début des années 1980 ont impulsé une dynamique partenariale (I). La plupart des professionnels et des responsables institutionnels s'accordent pour reconnaitre leur plus-value en termes d'expertise, de capacité d'action et de complémentarité des interventions. De nombreux sites offrent l'exemple de collaborations réussies, d'échanges inter-institutionnels réguliers et constructifs. Reste qu'en de nombreux lieux, les processus de restructuration à l'œuvre ne semblent pas bouleverser, du moins pas autant que la rhétorique partenariale ne le laisse supposer, les modes relationnels des différents acteurs. De sorte qu'il ne faut mésestimer, derrière ce qui relève de l'ordre du discours, la portée relative des changements introduits (II).

\section{I - L'institutionnalisation de la coproduction de la sécurité}

Le concept de coproduction s'appuie sur des préceptes mobilisateurs que l'on retrouve dans bien d'autres secteurs d'action publique: approche globale, partenariat, projet ou encore proximité. Ses promoteurs défendent une stratégie transversale, contextualisée, qui rassemble l'ensemble des acteurs locaux autour d'un agir de projet. Sur le plan opérationnel, les pouvoirs publics ont forgé de nouveaux outils méthodologiques pour stimuler et pérenniser la démarche partenariale (1). Là où les coopérations se sont véritablement affermies, les bénéfices de la coproduction ne sont plus à démontrer (2). 


\section{1 - Les outils de la coproduction}

Les dispositifs partenariaux se sont multipliés depuis vingt ans. Des structures interministérielles et collégiales sont apparues au plus haut sommet de l'État, soit pour rapprocher ses propres ministères (Conseil de sécurité intérieure, Conseil interministériel de prévention de la délinquance, Conseil interministériel des villes, Mission interministérielle de lutte contre la drogue et la toxicomanie, Délégation interministérielle à la ville, etc.), soit pour étendre la concertation aux acteurs périphériques (Conseil National des villes, Conseil national de l'aide aux victimes, etc.). C'est toutefois au niveau local que l'on retrouve le plus grand nombre de dispositifs opérationnels. À la suite des conseils communaux de prévention de la délinquance (CCPD) introduits au début des années 1980, les actuels conseils locaux de sécurité et de prévention de la délinquance, municipaux (CLSPD) ou intercommunaux (CISPD), réunissent, sous la présidence du maire ou du président de la structure intercommunale, le préfet, le procureur de la République, le président du conseil général, des représentants des services de l'État (police, gendarmerie, etc.), des représentants d'associations, d'établissements ou d'organismes œuvrant dans les domaines de la prévention, de la sécurité, de l'aide aux victimes, du logement, des transports collectifs, de l'action sociale ou des activités économiques (Art. D. 2211-2 du Code Général des Collectivités Territoriales). Selon l'article D. 2211-1 du CGCT, le CLSPD constitue le cadre de concertation sur les priorités de la lutte contre l'insécurité et de la prévention de la délinquance dans la commune. Il favorise l'échange d'informations entre les responsables des institutions et organismes publics et privés concernés, et peut définir des objectifs communs pour la préservation de la sécurité et de la tranquillité publiques. Il assure l'animation et le suivi du contrat local de sécurité [...]. Il est consulté sur la définition, la mise en ouvre et l'évaluation des actions de prévention de la délinquance prévues dans le cadre de la contractualisation entre l'État et les collectivités territoriales en matière de politique de la ville [...]. Le conseil local peut proposer des actions de prévention ponctuelles dont il assure le suivi et l'évaluation. Au $1^{\mathrm{er}}$ mai 2006, 867 conseils étaient recensés sur le territoire français. Depuis la loi du 5 mars 2007 relative à la prévention de la délinquance, leur installation est obligatoire dans les communes de plus de 10000 habitants et dans celles comprenant une zone urbaine sensible (Art. L2211-4 CGCT).

Parallèlement, un Conseil départemental de prévention de la délinquance, d'aide aux victimes et de lutte contre la drogue, les dérives sectaires et les violences faites aux femmes (CDP) rassemble la plupart des acteurs mentionnés précédemment, mais sous la présidence du préfet (Décret $\mathrm{n}^{\circ}$ 2006-665 du 7 juin 2006, art. 12). Ses missions sont diverses: faire des propositions utiles aux institutions publiques et privées en matière de prévention, élaborer un plan départemental de lutte contre la drogue et de prévention des conduites d'addiction, des programmes de prévention de la délinquance des mineurs et de lutte contre les violences faites aux femmes, susciter et encourager les initiatives en matière d'aide aux victimes ainsi que la mise en œuvre des travaux d'intérêt général, dresser le bilan chaque année de ces différentes actions. Ces instances partenariales coexistent parfois avec des Groupes locaux de traitement de la délinquance (GLTD) et des cellules de veille. Pilotés par la justice, les GLTD réunissent essentiellement le parquet, le maire et les forces de police pour agir dans des situations d'urgence et sur des sites névralgiques (Donzelot, Wyvekens, 2004). Ils visent un renforcement ponctuel de l'action judiciaire sur des zones affectées par une multiplication des délits, puis doivent disparaître ou être mis en sommeil dès l'amélioration de la situation. Les cellules de veille cherchent en amont à suivre l'évolution de l'insécurité dans les quartiers sensibles pour anticiper l'apparition de tensions ou de crises. Installées à l'initiative des maires ou des préfets, elles sont chargées d'identifier les problèmes, d'élaborer des 
procédures d'alerte rapide et de préparer la mobilisation des ressources nécessaires pour y faire face. Ces différentes enceintes de discussion accueillent le plus souvent des responsables institutionnels et des cadres intermédiaires. Certaines collectivités locales ont toutefois créé des espaces de dialogue associant plus étroitement les agents de terrain. Des élus de quartier, des policiers, des enseignants, des habitants, des éducateurs spécialisés, des assistantes sociales, etc., se rencontrent périodiquement au sein d' «instances territoriales de quartiers » à Rezé (44) ou de «cellules mensuelles de régulation territoriales » à Nantes (44). Cette présentation succincte des dispositifs partenariaux ne saurait prétendre à l'exhaustivité. De façon plus ou moins formelle, des réseaux d'acteurs aux contours variables se contruisent ici et là pour échanger sur des problématiques spécifiques (délinquance sexuelle, violences conjugales, lutte contre la toxicomanie, etc.). Entre autres exemples, des magistrats, des experts-psychiatres, des soignants et des travailleurs sociaux dialoguent au sein de « réseaux santé-justice ». Avec d'autres professionnels et responsables institutionnels (préfet, associations, police, etc.), ils élaborent des actions coordonnées dans le cadre de commissions départementales d'action contre les violences faites aux femmes, elles-mêmes divisées en sous-commissions thématiques (violences conjugales, harcèlement au travail, agressions sexuelles, etc.).

$\mathrm{Au}$ delà d'un simple rapprochement des acteurs, la doctrine de la coproduction vise la construction progressive d'une stratégie collective et partagée. Pour permettre aux professionnels d'avancer groupés, les pouvoirs publics ont imaginé de nouveaux outils méthodologiques qui s'inspirent de la technique contractuelle (Gautron, 2006). Depuis 1997, et en remplacement des contrats d'action de prévention pour la sécurité dans la ville (CAP), des contrats locaux de sécurité (CLS) transcrivent les orientations, le plan d'action et les engagements respectifs des acteurs. Ce dispositif contractuel doit être le point d'aboutissement d'une méthodologie rigoureuse (Gautron, 2008). La signature d'un CLS suppose la réalisation préalable d'un diagnostic local de sécurité (DLS). Les partenaires doivent mesurer l'ampleur et la nature des phénomènes de délinquance qui affectent leur territoire, l'importance du sentiment d'insécurité et apprécier l'adéquation des réponses apportées. Qualitatives autant que quantitatives, les données à recenser portent sur les caractéristiques locales de la délinquance, sur les lieux et les horaires de commission des infractions, sur le profil des délinquants, sur les incidents recensés par les établissements scolaires, les transporteurs et les bailleurs, sur les caractéristiques et les comportements des victimes, sur les attentes de la population, etc. Le diagnostic doit répertorier les différents moyens qui concourent à la sécurité, qu'ils soient préventifs ou répressifs, évaluer leur pertinence au regard du contexte local et les dysfonctionnements susceptibles d'en limiter l'impact. Sur la base des observations réalisées, les acteurs doivent s'accorder sur des objectifs, des priorités et un plan d'action. Une fois posés les éléments de diagnostic et le cadre général d'intervention, le contrat recense sous la forme de fiches-actions les programmes des différents partenaires, leurs calendriers de réalisation, les moyens engagés ainsi que des indicateurs de suivi et de résultat. Au 30 juin 2006, le ministère de l'Intérieur dénombrait 687 CLS, dont 195 intercommunaux. Cette formule contractuelle a été reconduite en 2006 sous la forme de CLS «nouvelle génération »'.

\footnotetext{
${ }^{1}$ Circulaire du 4 décembre 2006 relative à la prévention de la délinquance et à la préparation des contrats locaux de sécurité de nouvelle génération, NOR : INTK0600110C.
} 


\section{2 - Les atouts de la dynamique partenariale}

De nombreux travaux de recherche soulignent l'intérêt d'une approche partenariale et contractualisée. Cette démarche s'affirme comme une réponse à la fragmentation excessive des pouvoirs. L'interpénétration progressive du social et du pénal, associée à la dilution des catégories du public et du privé, a généré un processus d'hybridation des régulations dans le champ des politiques de lutte contre la délinquance (Gautron, 2006). Parce que les problèmes qu'ils ont en charge se cumulent ou se renforcent fréquemment, des interdépendances complexes se sont nouées entre acteurs. Leurs interventions se succèdent ou coexistent, se complètent ou au contraire se contredisent. Si ces interactions ne sont pas récentes, elles sont longtemps restées officieuses. L'attitude de l'État fut longtemps de les occulter, tant il était soucieux de préserver les symboles de la monopolisation du pouvoir de punir (Roché, 1998, 153). À partir d'une dissociation technique des problèmes et d'une division sectorielle des tâches, les institutions impliquées dans le traitement de la délinquance ont agi de façon verticale et autonome, selon leurs propres critères, valeurs et logiques d'action. Ce mode de fonctionnement s'est souvent traduit par des rivalités entre institutions, des logiques concurrentielles ou antagonistes, avec pour conséquences, diverses contradictions dans la conduite de l'action publique. A l'opposé, les dispositifs de coproduction reconnaissent et valorisent le phénomène d'hybridation des régulations. Ils permettent de sortir d'un modèle d'organisation éclaté entre de multiples rationalités sectorielles pour au contraire fédérer les initiatives, mutualiser l'expertise, les ressources et les savoir-faire des différents intervenants. Les réunions et contacts informels amorcent un dialogue entre acteurs sur leurs missions respectives. Ils favorisent de la sorte des logiques de décentrage, d'intercompréhension, une forme de réciprocation des perspectives (De Maillard, 2000a, 408). Les attitudes de réserve ou de méfiance s'estompent sous l'effet d'une meilleure connaissance, interconnaissance et reconnaissance entre membres du système d'action. Se constituent progressivement un langage commun, des codes partagés capables de produire une véritable synergie institutionnelle (De Maillard, 2000a, 411). Parce qu'ils intègrent et structurent les interdépendances entre secteurs d'intervention, les dispositifs de coordination assurent une meilleure polyvalence de l'action publique. Les acteurs développent des orientations communes, articulent leurs champs de compétences et construisent des actions conjointes.

Les dispositifs partenariaux enrichissent également l'expertise locale car ils facilitent la collecte et la comparaison d'informations dispersées et/ou difficiles d'accès (Ocqueteau, Frenais, Varly, 2002 ; Gautron, 2008). La police, la gendarmerie et l'institution judiciaire disposent de statistiques sur l'état de la délinquance, sur les personnes mises en cause, poursuivies et condamnées. Les établissements scolaires enregistrent des données sur le taux d'absentéisme, sur les incidents (vols, dégradations, violences, etc.), sur le nombre de procédures disciplinaires. La protection judiciaire de la jeunesse et les services de l'aide sociale à l'enfance possèdent des éléments d'information sur les mesures d'assistance éducative, sur le profil et la situation familiale des mineurs délinquants ou en danger. À l'identique, les bailleurs sociaux, les associations, les centres socioculturels ou encore les sociétés de transport en commun possèdent une part des informations utiles. Plus qu'un simple recensement des données statistiques, les évaluations et diagnostics locaux permettent d'inclure dans l'analyse des données qualitatives jusqu'alors peu prises en compte : contenu des mains courantes, courriers envoyés aux élus, appréciations de la population et des associations de quartier, etc. Ces outils génèrent une connaissance plus fine des caractéristiques locales de la délinquance (nature, localisation, etc.). En confrontant leurs expertises respectives, les acteurs développent progressivement un cadre d'analyse commun. Il leur est alors possible d'établir des «cibles » et des programmes d'action adaptés aux 
caractéristiques locales de la délinquance. L'évaluation in itinere ou a posteriori permet ensuite de contrôler le degré de conformité des interventions au regard du plan d'action initial, de détecter les effets pervers induits par l'action publique et de la réorienter en continu. Elle incite les acteurs à opérer des retours réflexifs sur leurs pratiques, à analyser de façon critique leurs modes de fonctionnement.

La contractualisation assure quant à elle la transparence et la prévisibilité des engagements. Elle stabilise et sécurise les relations institutionnelles (Donzelot, Wyvekens, 2004). Sauf de rares exceptions ${ }^{2}$, les C.L.S. ne peuvent être considérés comme de véritables contrats au sens juridique du droit privé ou du droit administratif. Leurs énoncés sont le plus souvent imprécis et ne permettent pas d'engager, en cas de manquement, la responsabilité des signataires. Malgré l'imprécision des engagements et la faiblesse du cadre juridique, les négociations aboutissent toutefois à des accords qui sont généralement tenus. Le non-respect d'un engagement serait d'un coût trop élevé car il priverait son auteur du soutien de ses partenaires lors de la concrétisation des accords ou à l'occasion d'autres conventions (Gaudin, 2001). Ces contrats forment bien la loi des parties, mais ils tirent leur force des relations d'ordre politique et institutionnel qu'ils permettent d'organiser, et qui leur confèrent une sorte d'objectivité sociologique, sans doute davantage que leur inscription dans l'ordre juridique (Marcou, 1997, 114). Cet outil de programmation donne aux acteurs la possibilité d'anticiper les actions engagées par leurs partenaires, d'en vérifier le respect et de prévoir à moyen terme les financements dont ils bénéficieront pour la conduite de leurs propres opérations.

Ces différents atouts expliquent les avancées de la dynamique partenariale depuis le milieu des années 1980. Plusieurs études évoquent au sujet des CLSPD et des CLS un partenariat plus ouvert (Gorgeon, Estèbe, Léon, 2000 ; Donzelot, Wyvekens, 2004 ; Le Goff, 2003 ; De Maillard, 2008). Dans plusieurs sites, un système collectif d'échanges s'est progressivement structuré. Les différents intervenants apprennent à s'écouter mutuellement, à dépasser des logiques d'imputation visant à attribuer aux autres la responsabilité des déficiences de l'action publique. Outre l'implication croissante de l'éducation nationale, de la police et de la justice, le réseau d'action s'est ouvert à de nouveaux participants : commerçants, bailleurs, pompiers, transporteurs, etc. Selon une enquête statistique réalisée en 2002 par le ministère de l'Intérieur, 61\% des CLS comportaient à l'époque des engagements des transporteurs publics, $80,8 \%$ des bailleurs sociaux et $41,2 \%$ des acteurs économiques (chambres de commerce et de l'industrie, entreprises commerciales, etc.) ${ }^{3}$. Dans l'agglomération nantaise, la Chambre de commerce et de l'industrie a mis en place des ateliers de la sécurité où les commerçants sont amenés à dialoguer avec la police. Quelques expériences illustrent une implication plus affirmée des acteurs privés de sécurité (Mothes, Novaro, Pasinetti, 2005). À Toulouse, ces derniers collaborent avec les médiateurs sociaux et la police nationale pour renforcer la sécurité dans le métro. Des représentants de sociétés de gardiennage assistent aux réunions mensuelles du comité de suivi du CLS de Noisy-le-Grand, etc. Progressivement, les différents intervenants s'accordent sur des orientations générales, s'approprient de nouvelles méthodes de travail et redéfinissent leurs modes d'intervention dans une perspective directement opérationnelle (Le Goff, 2003). De sorte que les CLS s'écartent de la logique essentiellement représentative des CCPD, logique qui cantonnait le partenariat à des échanges d'informations

\footnotetext{
${ }^{2}$ Il a été jugé à propos d'un CLS qui fixait de manière détaillée ses modalités de mise en œuvre et de financement qu'il avait une portée contractuelle. Un tiers peut poursuivre l'annulation des actes qui en sont détachables, au nombre desquels la décision de le conclure, à la condition que les stipulations de ce contrat soient de nature à léser le requérant dans ses intérêts de façon suffisamment certaine et directe (T.A. Paris, 26 décembre 2000, M. Puzin, req. N9912265/3).

${ }^{3}$ Source : Ministère de l'Intérieur, non publiée.
} 
et de propositions, sans toutefois aboutir à l'élaboration d'un projet partagé (Donzelot, Wyvekens, 2004).

Bien d'autres dispositifs de concertation démontrent que des mécanismes d'apprentissage collectif sont à l'œuvre. Dans plusieurs départements, les professionnels de la justice et de la santé discutent et s'accordent sur les moyens d'articuler la loi, la clinique et l'éthique au sujet des expertises psychiatriques et des soins pénalement obligés (Gautron, 2009). Alors qu'il s'agit d'une question lourde d'enjeux d'un point de vue juridique et déontologique, certains acteurs acceptent d'échanger des informations nominatives concernant les difficultés posées par tel ou tel individu, telle ou telle famille. En Loire-Atlantique, un groupe de travail composé de professionnels du Conseil général, de l'éducation nationale, des municipalités de l'agglomération nantaise et de la PJJ a rédigé en 2006 une "Charte relative à l'échange d'informations au sein des instances locales de partenariat ». Visant à encadrer les pratiques jusqu'alors informelles et au cas par cas de « secret partagé », ce texte a été relativement bien accueilli par les acteurs de terrain. Les débats inter-institutionnels préalables et sa rédaction à plusieurs mains ont permis de préserver les règles déontologiques et les logiques professionnelles des uns et des autres. Une définition stricte de la finalité et des modalités du partage d'informations, limité aux éléments utiles à l'accompagnement social des familles de mineurs en difficulté, a eu pour effet d'atténuer les réserves initiales des professionnels, notamment celles des travailleurs sociaux. Si ces échanges sont loin d'être systématiques et réguliers, les partenaires locaux communiquent plus fréquemment sur des situations individuelles dans les cellules de veille ou autres dispositifs de concertation à Nantes, Rezé et Saint-Herblain.

Ces résultats prometteurs expliquent la promotion continue de la doctrine de la coproduction depuis une vingtaine d'années. Son succès est tel que chaque acteur civil et chaque agent des multiples cléricatures semble aujourd'hui avoir pour mission de rassembler autant de partenaires que possible pour mener à bien les activités qui lui ressemblent et à travers lesquelles il s'affirme et se donne à voir. Ce rassemblement dit "coopératif » est alors la réponse privilégiée à tout dysfonctionnement, à tout éclatement et à toute rupture. Les tentatives de développement ou simplement d'installation d'une dynamique de travail, d'appropriation d'un territoire, de réponse à une question, sont très souvent jugées à l'aune de leur capacité à s'inscrire dans un partenariat, et vouées rapidement aux gémonies et à l'échec lorsqu'elles ne mobilisent pas autour d'elles ou de leur géniteur des hommes et des groupes associés, dont la seule apparition en légitime le caractère opératoire, utile et publiquement correct (VIEILlE-GrosJEAN, in DHUME, 2001, 91). En 2002, le changement de majorité politique n'a donc pas donné lieu à une suppression des dispositifs partenariaux installés par la gauche plurielle à la fin des années 1990. Outre la généralisation des CLSPD dans les communes de plus de 10000 habitants, les contrats locaux de sécurité ont été reconduits en 2006 sous une forme relativement similaire d'un point de vue méthodologique (diagnostic, objectifs, plan d'action, évaluation). De nouveaux acteurs sont par ailleurs incités à rejoindre les dispositifs de coordination et/ou à participer à la réalisation du DLS. De façon à associer l'ensemble des acteurs impliqués dans la chaîne de sécurité et de prévention, la circulaire du 4 décembre 2006 évoque notamment les hôpitaux, la poste, les entreprises publiques (EDF, etc.), les huissiers ou encore les services fiscaux. Il est vrai que lorsque de véritables échanges prennent forme, les enceintes partenariales facilitent un ajustement des représentations, valeurs et modes opératoires des différents acteurs. Les collaborations demeurent néanmoins très variables sur le terrain. Entre la philosophie affichée par la doctrine de la coproduction et les résultats concrets de son implémentation, l'écart est souvent important et les contradictions nombreuses. 


\section{II - Une coproduction imparfaite}

La doctrine de la coproduction présuppose une inclination naturelle des professionnels à s'investir dans les dispositifs de concertation. Or, il ne suffit pas de décréter le partenariat pour qu'il se traduise dans les faits. Une approche diachronique démontre que les dispositifs de coordination introduits depuis le début des années 1980 n'ont modifié qu'à la marge les modes relationnels des différents acteurs. De son côté, l'État promeut une version tronquée du principe de coproduction tant il s'efforce de maintenir, sinon d'accentuer, son leadership dans le champ de la sécurité (1). Dans leur ensemble, les professionnels s'affranchissent difficilement des divergences de représentations, de valeurs, de normes d'action et d'intérêts. Suivant leurs appartenances professionnelles et/ou institutionnelles, ils entendent défendre leurs visions respectives de ce qui fait problème, de ce qui est efficace et rationnel. Sans donner lieu à des oppositions frontales, ces désaccords alimentent des attitudes de réserve, de méfiance ou de retrait. Les échanges restent trop souvent sporadiques et les collaborations ponctuelles, à l'opposé de rapports de complémentarité durables et réguliers (2).

\section{1 - Un positionnement ambivalent de l'État}

L'idée même de coproduction tend à valider l'hypothèse d'une perte de centralité de l'État dans la conduite des politiques de lutte contre la délinquance. Cette nouvelle méthode d'action publique traduirait le passage d'un État-providence interventionniste et propulsif (Chevallier, Morand, 1991) à un État modeste (Crozier, 1997). Celui-ci n'imposerait plus le changement social par le biais d'un droit autoritaire mais fédèrerait les initiatives, initierait des coopérations, expérimenterait de nouvelles modalités d'action, informerait, conseillerait et coordonnerait l'ensemble des interventions. Pour qualifier ce repositionnement, les chercheurs parlent tour à tour d'État animateur (Donzelot, Estèbe, 1994), pédagogue (Gaudin, 1999, 68), réflexif (Ost, van de Kerchove, 2002, 144), incitateur (Morand, 1999, 159) ou encore superviseur (Papadopoulos, 1995, 13). Celui-ci chercherait à barrer plus qu'à ramer, à guider plus qu'à faire lui-même (Crawford, 2001b, 18). Il institutionnaliserait à cet effet des programmes relationnels lui confèrant un rôle d'entremise (Ost, van de Kerchove, 2002, 144). Comme dans d'autres sphères d'action publique, le paradigme de la gouvernance s'impose (Roché, 2004 ; Gleizal, 2003 ; De Maillard, 2003 ; Hassid, 2005 ; Dupont, 2008) pour décrire cet art complexe consistant à piloter des agences, des institutions et des systèmes multiples, autonomes sur le plan opérationnel les uns par rapport aux autres, mais en même temps structurellement couplés entre eux par le biais de formes diverses d'interdépendance réciproque (Papadopoulos, 2001, 167). L'idée de direction et de pilotage étatique est bien présente, sans exagérer toutefois la structuration hiérarchique du pouvoir au profit d'un acteur central et dominant (Le Galès, 1998 ; Baron, 2003). Dans le champ de la sécurité, cette conversion des méthodes d'action publique n'accompagne pas une véritable perte de centralité de l'État. Une entrée par les discours montre que ce dernier développe une conception ambivalente de la coproduction (A). Paradoxalement, la méthode contractuelle et partenariale semble instrumentalisée pour restaurer, sinon affermir, son autorité (B).

\section{A - Une conception équivoque du principe de coproduction}


La plupart des textes ou discours publics qui se référent aux politiques partenariales et contractualisées ne ravalent pas l'État au rang de partenaire ordinaire. Les responsables politiques lui attribuent au contraire une fonction stratégique, celle de sauvegarder l'intérêt général, de corriger les déséquilibres économiques et sociaux, de maintenir la cohésion et la solidarité nationale. Ils le présentent comme un tiers régulateur, une instance capable, par sa position d'extériorité et de supériorité par rapport aux intérêts en présence, de ramener la diversité à l'unité, l'hétérogénéité à l'homogénéité, le désordre à l'ordr» (Chevallier, in Arnaud, 1999, 169). L'État entend conserver un rôle prépondérant dans la définition des objectifs et des priorités d'action publique (Papadopoulos, 1995). Les politiques se construisent à deux niveaux successifs. Dans un premier temps, l'État formule les objectifs et les orientations de la politique qu'il envisage. Dans un second temps, il élabore des normes et des dispositifs décentralisés visant l'émergence de solutions déductives respectueuses des particularités locales. Par ce biais, l'État concilie la nécessité de définir de grandes orientations politiques nationales avec une marge d'initiative indispensable des gouvernés (Gleizal, 2003).

Les politiques pénales sont une parfaite illustration de cette ambivalence. Parce que la légitimité de l'État dépend de sa capacité à rassurer et à protéger (Roché, 1998), la consécration du principe de coproduction coexiste avec la réaffirmation, bien qu'allusive, de son monopole pénal. Texte fondateur de la dynamique partenariale au début des années 1980 , le rapport Bonnemaison avait pris soin de rappeler que la sécurité des citoyens est de la responsabilité de l'État (Commission des maires sur la sécurité, 1982, 11). Dans la même veine, la loi n95-73 du 21 janvier 1995 précise que la sécurité est un droit fondamental et l'une des conditions de l'exercice des libertés individuelles et collectives. L'État a le devoir d'assurer la sécurité en veillant, sur l'ensemble du territoire de la République, à la défense des intérêts nationaux, au respect des lois, au maintien de la paix et de l'ordre public, à la protection des personnes et des biens. La circulaire du 28 octobre 1997 relative aux contrats locaux de sécurité indique quant à elle que la sûreté est pour l'édifice de la République le socle nécessaire à l'exercice de toutes les libertés. C'est le premier droit des citoyens. C'est la mission première de l'État. On retrouve ici l'imprégnation historique d'un État jacobin présenté comme premier et unique responsable du traitement pénal de la délinquance. Par la référence à des concepts forts et universalisants comme la République, la sécurité intérieure, la sûreté, la citoyenneté ou encore la liberté, l'Etat manifeste sa volonté de maintenir la primauté de son intervention, de se poser comme initiateur et garant de l'action pénale. La substance du principe de coproduction semble dès lors minorée lorsque l'on aborde les questions de répression des infractions. L'ensemble des discours, circulaires et textes législatifs qui circonscrivent les politiques locales de sécurité précisent que les maires et autres partenaires contribuent, sont associés ou participent à l'action publique dans le champ pénal. Nous sommes loin de la coproduction, le préfixe co signifiant avec, ensemble. Lorsque l'on dépasse cette dimension intellectuelle et cognitive pour observer les pratiques, cette hypothèse se vérifie pleinement.

\section{B - La subordination des projets locaux aux exigences de l’État}

Comme dans d'autres champs d'action publique, la propension de l'État à organiser son propre décentrement peut être appréhendée comme une manière de récupérer son pouvoir, voire d'assurer son expansion selon des modalités nouvelles (Arnaud, 1999, 160). Si les 
nouveaux registres d'action issus de la doctrine de la coproduction le parent des vertus de l'ouverture et de l'humilité, ils n'induisent pas une égalisation des rapports qu'il entretient avec ses partenaires. Dans le processus contractuel, l'État détient des pouvoirs que ne possède aucun autre acteur, pouvoirs qui lui assurent une position de leadership. Il concentre l'essentiel des prérogatives d'impulsion, d'expertise, de réglementation, de financement et de contrôle (Gaudin, 1999). Conçues par les administrations centrales, les procédures contractuelles font l'objet d'un encadrement formel et méthodologique important (BodyGendrot, Duprez, 2001). Dans une logique de prêt-à-porter plus que de sur mesure (De Maillard, 2000a, 130), des circulaires ministérielles définissent précisément leur architecture, les différentes étapes de la démarche, les partenaires légitimes, les découpages thématiques souhaitables, le calendrier. La définition centralisée du cadre des coopérations préserve la dissymétrie des pouvoirs entre l'État et ses partenaires. En canalisant l'offre de négociation, ce dernier leur impose des modèles de conduite et réduit corrélativement leurs marges de manœuvre. Ce rôle d'initiateur, sans garantir une maîtrise absolue de la démarche contractuelle, confère aux autorités centrales un rôle de premier plan dans la discussion des objectifs et la conduite de l'action (Gaudin, 1999 ; Epstein, 2008). Du fait de la production centralisée de règles formelles, de schémas relationnels et de cadres d'intervention, la technique contractuelle induit une normalisation et une homogénéisation des pratiques locales (De Maillard, 2000a).

L'État dispose également du pouvoir de labelliser les acteurs qu'il souhaite ou non associer à sa politique. De cette opération de sélection découle un partenariat majoritairement interinstitutionnel, au sein duquel prédominent ses propres représentants. Si l'on suit l'ordre de présentation des acteurs sollicités dans la circulaire du 28 octobre 1997 relative à la mise en œuvre des contrats locaux de sécurité, on constate qu'une hiérarchie subtile organise les relations entre partenaires. Ces contrats sont élaborés par le préfet, le procureur de la République et le (ou les) maires. Les autres partenaires se voient confier un rôle plus limité. Le préfet veillera à associer aux réflexions préparatoires à la négociation des contrats tous les acteurs institutionnels et sociaux dont la participation [...] est nécessaire à la conduite d'une vaste et réelle concertation. Comme au début des années 1990, les quatre acteurs régaliens représentés par le préfet, le parquet, la police et la gendarmerie nationales forment l'épicentre du système d'action (Thoenig, 1994). Le cœur du dispositif partenarial réunit généralement le directeur de cabinet du préfet, le directeur départemental de la sécurité publique, un représentant de la ville (maire, élu, chargé de mission) et, dans une moindre mesure, un représentant du parquet (Le Goff, 2003 ; Gorgeon, Estèbe, Léon, 2000 ; Sina, 2002). Bien que consultés par les acteurs décisionnels et associés à la mise en œuvre de certains types d'intervention, les autres partenaires (transporteurs, bailleurs sociaux, associations, commerçants, travailleurs sociaux, etc.), sont souvent rejetés à la marge du processus de décision.

Une hiérarchie subtile ordonne ensuite les relations entre les services de l'État et ceux des collectivités locales. À l'inverse des dispositifs introduits au début des années 1980, qui ont permis aux élus locaux d'expérimenter en toute autonomie des politiques municipales de prévention et de tranquillité publique, la tutelle des administrations régaliennes s'est considérablement renforcée depuis la seconde moitié des années 1990 (Gleizal, 1995). Sans quelque paradoxe avec l'inscription d'un pilotage municipal des CLSPD et des CLS, la loi réaffirme périodiquement que le préfet anime et coordonne la prévention de la délinquance et de l'insécurité (art. 6, loi du 21 janvier 1995). Ce phénomène de recentralisation semble s'accentuer depuis 2006. Certes, la circulaire du 4 décembre 2006 relative aux CLS de «nouvelle génération » qualifie l'ensemble des partenaires de co-contractants. Le nouvel article L2211-4 du CGCT, issu de la loi du 5 mars 2007, consacre le rôle pivot du maire en 
matière d'animation et de coordination des politiques de prévention. Toutefois, le nouvel article L2215-2 du même code dispose que les actions de prévention de la délinquance conduites par les collectivités territoriales ne doivent pas être incompatibles avec le plan de prévention de la délinquance arrêté par le représentant de l'État dans le département. L'autonomie des élus locaux en ressort amoindrie par rapport aux politiques de prévention et de tranquillité publique des années 1980. Les marges de manœuvre concédées aux élus locaux se réduisent davantage lorsqu'il est question de répression. Ce sont en réalité des dispositifs spécifiques, placés sous la coupe des administrations régaliennes, qui adaptent localement les politiques pénales et de sécurité définies par les administrations centrales. Sous la double présidence du préfet et du procureur de la République, un comité départemental de sécurité (CDS) composé des seuls services de l'Etat anime, coordonne et met en œuvre les politiques de sécurité intérieure dans le département. L'article L2215-2 du CGCT, introduit en 2007, se contente d'indiquer que le représentant de l'État dans le département associe le maire à la définition des actions de lutte contre l'insécurité. Les stratégies territoriales de la police et de la justice restent donc très peu discutées dans le cadre contractuel (Le Goff, 2004). La définition de la politique pénale ne donne lieu à aucune forme de concertation entre le ministère public et les acteurs extra-judiciaires. Au sujet des politiques de lutte contre la toxicomanie, une circulaire du 9 mai 2008 adressée aux parquets précise même que le Conseil départemental de prévention doit prendre en compte les priorités résultant des décisions judiciaires en réponse à la toxicomanie ${ }^{4}$. Le procureur de la République, en sa qualité de viceprésident, est simplement invité à exposer sa politique d'action publique en matière de lutte contre l'usage de drogue. Deux logiques séparées se maintiennent : une logique contractuelle, consacrée pour une grande part aux initiatives municipales dans le domaine de la tranquillité publique relayées par celles d'autres partenaires; une logique sectorielle, interne à la police et à la justice, qui n'interfère qu'à la marge avec la première (Gorgeon, Estèbe, Léon, 2000, 240).

La surface financière de l'État lui permet enfin de se placer au centre des négociations locales. À la fin des années 1990, il a usé de ses capacités contributives pour inciter les élus locaux à signer des CLS, et ce malgré l'absence initiale de crédits spécifiques. Des élus ont accordé leur signature pour recruter des agents locaux de médiation sociale (ALMS) et des adjoints de sécurité (ADS), financés à 85\% par l'État dans le cadre du programme « emplois-jeunes » (Le Goff, 2002a). Ce dernier leur a également promis d'accroître les effectifs policiers et judiciaires sur leurs territoires, de créer des antennes de police ou des maisons de justice. D'autres crédits, qui ne se destinaient pourtant pas aux programmes de lutte contre la délinquance, ont été utilisés comme instruments de marchandage. Dans plusieurs villes, les maires ont accepté de signer des CLS ou des contrats de ville suite à l'engagement préfectoral de financer des projets d'aménagement urbain ou la construction de logements sociaux (Le Goff, 2002b ; De Maillard, 2000a). Les services de l'État fixent par ailleurs des critères d'allocation qui s'imposent largement à ceux qui souhaitent être financés. Cette pression financière s'accentue depuis la création du fonds interministériel de prévention de la délinquance (FIPD) en 2006. Deux circulaires du 21 février 2008 et du 23 janvier $2009^{5}$ précisent que les subventions doivent abonder les programmes qui s'inscrivent dans les priorités définies par le Comité interministériel de prévention de la délinquance et déclinées dans les plans départementaux de prévention, au premier rang desquelles l'installation de

\footnotetext{
${ }^{4}$ Circulaire de la DACG 2008 - 11 G4/ du 9 mai 2008 relative à la lutte contre la toxicomanie et les dépendances, NOR : JUSD0811637C.

${ }^{5}$ Circulaire NOR INTK0800042C du 21 février 2008 relative aux orientations du fonds interministériel de prévention de la délinquance pour 2008; Circulaire NOR INTK0900017C du 23 janvier 2009 relative aux orientations du fonds interministériel de prévention de la délinquance pour 2009. [En ligne], URL [http://www.sgcipd.interieur.gouv.fr]. Consulté le 2 février 2009.
} 
caméras de vidéosurveillance dans les espaces publics et leur raccordement aux services de police (28 M€, soit environ 75\% des sommes affectées au FIPD pour l'année 2009). Pour ne pas perdre des ressources ou au contraire les amplifier, les acteurs sont tentés d'adapter leurs projets aux conditions posées par les préfectures. La mairie de Nantes, dont le système de vidéosurveillance concerne uniquement le contrôle de la circulation routière, affronte ainsi quelques pressions préfectorales visant l'utilisation des caméras pour surveiller l'espace public. Si l'équipe municipale reste réticente à l'idée de généraliser la vidéosurveillance, des négocations sont en cours concernant la transmission des enregistrements à la police. Sur l'ensemble du territoire national, de nombreux élus parlent donc de contrats léonins ou d'adhésion, de produits de l'État destinés au financement de ses propres actions (De Maillard, 2000a). Les finances locales complètent effectivement les budgets des ministères de la Justice et de l'Intérieur. Les collectivités territoriales participent à la fourniture d'équipements, construisent ou mettent à disposition des locaux pour des antennes de police ou des maisons de la justice et du droit.

$\mathrm{Au}$ travers d'un jeu subtil intriquant pédagogie et injonction, l'État ambitionne donc d'assujettir les pratiques locales à sa propre rationalité. La technique contractuelle lui permet d'expérimenter de nouveaux rapports d'autorité auprès de ses partenaires dès lors qu'elle vise à faire entrer les collectivités locales dans les cadres qu'il a définis, avec les moyens d'action qu'il a retenus et selon les processus qu'il a arrêtés (De Maillard, 2000a). Comme l'écrivait déjà J. Chevallier en 1986, le cadre contractuel ne donne aux unités périphériques que l'illusion de la liberté : ces contrats ne mettent pas en présence des partenaires égaux, l'Etat dispose de moyens de pression, directs ou indirects, tels que les unités périphériques sont obligées de passer, bon gré mal gré, sous ses fourches caudines. La technique contractuelle est un vecteur privilégié d'unification et d'intégration de l'appareil [administratif] dans la mesure où elle permet d'obtenir la collaboration active des unités périphériques à l'exécution des priorités fixées par le niveau central, sans que celui-ci ait besoin de recourir à la contrainte: les unités périphériques sont amenées, pour obtenir le concours financier de l'État, à adhérer à sa propre rationalité, à intérioriser ses normes et à reprendre ses objectifs à leur propre compte (cité in De Maillard, 2000a, 135).

On se trouve dès lors face à un étrange paradoxe. Les politiques engagées à partir des années 1980 constituent à la fois l'antithèse et le décalque de celles qui les ont précédées. Avant que le principe de coproduction des politiques de lutte contre la délinquance n'émerge, leur élaboration et leur mise en œuvre étaient présentées et légalisées comme des attributs exclusifs de l'État. En pratique, ce monopole n'était que relatif, diverses régulations infraétatiques ayant résisté aux manœuvres centralisatrices des gouvernements successifs (Gautron, 2006). Plusieurs acteurs périphériques, parmi lesquels les notables locaux et les associations, ont su conserver un poids non négligeable au fil du temps, tant dans le domaine du règlement pénal des différends que dans celui des fonctions de police ou de prévention. Au-delà, les transformations de la philosophie pénale dominante à l'aube du $\mathrm{XX}^{\mathrm{e}}$ siècle, valorisant les actions de prévention et de traitement curatif de la délinquance, ont donné lieu à une interpénétration des champs du social et du pénal, et corrélativement du privé et du public. Malgré ce processus d'hybridation des régulations en matière de prévention et, dans une moindre mesure, de répression, l'État s'est évertué à protéger les symboles de la monopolisation du pouvoir de punir (Roché, 1998). À l'inverse, les dispositifs d'action nés dans les années 1980 ont reconnu et valorisé ce phénomène d'hybridation, tout en protégeant la prééminence étatique au sein du système d'action. En réalité, les déformations qui affectent le modèle français de lutte contre la délinquance sont donc essentiellement normatives. L'ambition initiale des pouvoirs publics consistait pourtant à enrayer, par la diffusion des approches partenariales et contractuelles, la crise d'un système politico-administratif 
centralisé et sectorisé. En conservant une logique tutélaire, l'État reproduit les insuffisances de ce modèle jacobin. Malgré l'ampleur des ressources qu'il est susceptible de mobiliser, il ne parvient que partiellement à imposer ses décisions (Gautron, 2006). Sa prééminence sur la scène locale dépend de sa capacité à se présenter, auprès de ses interlocuteurs, comme un ensemble de services poursuivant, dans une logique d'alliance, les mêmes objectifs. Or, au lieu de se coaliser pour peser face aux acteurs périphériques, les services étatiques défendent chacun leurs propres conceptions et leurs propres intérêts. Ces postures défensives ne sont pas le propre des services étatiques. Elles parcourent l'ensemble du réseau d'action. La dynamique partenariale s'affronte à des obstacles qu'elle ne parvient pas à surmonter.

\section{2 - Les écueils de la dynamique partenariale}

Qu'ils agissent en amont, pendant ou en aval du processus pénal, les acteurs impliqués dans la politique criminelle ont des perceptions, des analyses, des valeurs et des normes d'action qui diffèrent suivant leur appartenance institutionnelle et/ou professionnelle. Réservés sinon méfiants vis-à-vis de cette nouvelle démarche coopérative (A), ils investissent peu les enceintes partenariales (B). Ils s'associent et se sollicitent ponctuellement, notamment dans des situations d'urgence. Sur le long terme, leurs échanges restent toutefois sporadiques ou s'essoufflent au fil du temps. Nombre d'entre eux se contentent d'investir les procédures contractuelles sur un mode utilitariste, avec pour seule préoccupation la maximisation de leurs parts respectives de crédits et la défense de leurs pratiques habituelles $(C)$.

\section{A - Les réserves et résistances des acteurs}

Les divergences de représentations, de valeurs, de normes d'action et d'intérêts prédominent sur la scène locale. Elles alimentent diverses oppositions qui, sans être nécessairement frontales, entravent l'émergence de rapports de complémentarité. Au sein même de l'État, le corporatisme sectoriel résiste. Ses tentatives pour moderniser son architecture institutionnelle, décloisonner ses services et favoriser une approche transversale des problèmes ne lui ont pas permis de s'affranchir des rivalités institutionnelles, que ce soit au niveau central ou déconcentré. S'ils ne récusent pas l'intérêt d'une approche multidimensionnelle, ses agents perçoivent encore l'interministérialité comme une potentielle menace. Ils craignent une restriction de leurs compétences respectives, ne serait-ce qu'en raison du droit de regard qu'ouvrent les échanges inter-institutionnels sur leurs pratiques. L'implication dans les dispositifs de concertation est alors souvent conditionnée par la poursuite d'un strict respect de leurs domaines de compétence, voire d'une extension de leur champ d'action. De sorte que le préfet ne parvient pas à fédérer les services déconcentrés pour arrêter une stratégie commune (Estèbe, 1999 ; Robert, 1999). La justice, l'éducation nationale et dans une moindre mesure la police et la gendarmerie échappent à son pouvoir de direction. Son autorité est plus théorique qu'effective sur les autres services de l'État. Les administrations centrales maintiennent une forte tutelle sur leurs échelons territoriaux et tentent souvent de contourner le pouvoir du préfet au niveau local, notamment par un contrôle minutieux de l'affectation des crédits théoriquement déconcentrés. Dans les enceintes de discussion, les échanges entre services déconcentrés et préfecture sont traditionnellement mauvais, placés sous le signe d'un procès permanent et réciproque (Estèbe, 1999, 186). Les premiers dénoncent le caractère autoritaire du leadership préfectoral. Ils se méfient de la logique clientéliste induite par la 
proximité des préfets et des élus locaux. Ils refusent d'être subordonnés à un préfet qui, bien que représentant local de l'ensemble des ministères, dépend hiérarchiquement du ministère de l'Intérieur. Entre autres exemples, la cohabitation du préfet et du procureur au sein des dispositifs de pilotage n'est pas toujours évidente. Pour contrer le leadership préfectoral, certains procureurs ont fait pression pour obtenir «leur» instance de concertation sous la forme de GLTD (Le Goff, 2002b). Des dissensions vont jusqu'à opposer des services relevant d'une même administration, notamment au sein du ministère de la Justice (Dubouchet, Berlioz, 2001). Le procureur de la République n'est pas en mesure de s'imposer comme chef de file des différents intervenants judiciaires. Il ne peut s'engager au delà de la politique pénale car les magistrats du siège, les agents de la protection judiciaire de la jeunesse et de l'administration pénitentiaire évoquent selon les cas leur indépendance, leurs références et leur éthique professionnelles pour refuser d'être subordonnés au ministère public. Au regard de ces différents éléments, la lecture de l'État local apporte une sorte de preuve par l'absurde, celle de l'inexistence d'une entité qui pourrait porter ce nom - d'État local. Tout se passe comme si, en effet, toutes les tentatives pour coordonner, fusionner, unifier autour d'un objectif territorial commun, dans le cadre d'une procédure contractuelle, les services de l'État auvrant sur le territoire se soldaient non pas par un échec flagrant mais par une dispersion plus grande encore (Estèbe, 1999, 221).

Ce constat vaut pour les relations qui s'établissent entre collectivités locales. Les échanges entre communes, départements et régions dans le champ de la sécurité et de la prévention n'ont pas mis un terme aux conflits qui les opposent fréquemment. Ces oppositions ne s'expliquent pas seulement par les affiliations partisanes des élus. Dans de nombreuses agglomérations, dont Nantes métropole, les CLS ont réuni des maires dont les sensibilités politiques sont différentes. Les clivages idéologiques en matière de sécurité sont par ailleurs moins prégnants que par le passé (Mouhanna, 2005). Nombre de collectivités locales s'inscrivent par contre dans des logiques concurrentielles. Les maires des grandes villes, qui recouvrent une part importante de la population départementale, entretiennent souvent des relations difficiles avec les Conseils généraux. En participant aux dispositifs de concertation, les élus départementaux craignent d'être placés sous la tutelle des maires, notamment lorsqu'il s'agit de députés-maires d'agglomérations importantes. Les conflits partisans et les concurrences entre collectivités locales expliquent tout autant le succès relatif de l'intercommunalité de projet (Le Goff, 2002a; De Maillard, 2000a). Les communes périphériques reprochent aux maires des villes-centre d'imposer leur leadership dans les structures intercommunales. Les collaborations restent dès lors minimales. Chaque municipalité instrumentalise la démarche intercommunale pour développer ses ressources propres, sans souci de cohérence avec les interventions de ses partenaires. Si les collectivités territoriales s'affrontent, elles se coalisent bien souvent contre l'autorité préfectorale, soupçonnée d'ingérence dans les affaires locales. De nombreux élus reprochent aux préfectures d'empiéter sur leurs domaines de compétence et de ne voir en eux qu'une source supplémentaire de financements à destination des services de l'État. Avant même la création des CISPD et des CLS, plusieurs municipalités de l'agglomération nantaise se sont ainsi réunies dans une instance intercommunale de concertation, pour échanger sur les questions de prévention de la délinquance, mais surtout pour avancer groupées face au préfet.

Plus fondamentalement, ce sont les divergences de représentations, de normes et de logiques d'action qui prévalent au sein du système d'acteurs. Les professionnels portent généralement un regard critique sur les activités des institutions avec lesquelles ils collaborent. Plutôt que d'engager de véritables retours réflexifs sur leurs pratiques respectives, de façon à comprendre comment chacun contribue à la détérioration de la sécurité ou au manque d'efficacité de l'action publique, beaucoup s'enferment dans une dénonciation inlassable des 
carences de leurs partenaires (Donzelot, Wyvekens, 2004). La plupart se retrouvent pour repousser le leadership municipal. La priorité des élus ne serait pas la conduite d'actions concrètes et efficaces, mais une politique de communication destinée à satisfaire les revendications de leur électorat. Pour renforcer leur position sur l'échiquier politique local, ils adopteraient des pratiques clientélistes, notamment le financement d'institutions ou d'associations qui leur sont proches (De Maillard, 2000a). Ces suspicions sont particulièrement fortes chez les magistrats et les travailleurs sociaux, qui craignent d'être instrumentalisés par les municipalités (Wyvekens, 1997 ; De Maillard, 2000a ; Mouhanna, Ackermann, 2001). Différents clichés ou préjugés alimentent encore ces réserves et reproches réciproques. Les policiers dénoncent le laxisme des magistrats, notamment de la jeunesse, qui libèreraient les délinquants aussitôt interpellés. L'angélisme des travailleurs sociaux est souvent pointée du doigt, que ce soit par les magistrats ou les policiers (Tiévant, 2002 ; Boucher, 2007). À l'opposé, les travailleurs sociaux, les professionnels de la santé et dans une moindre mesure les enseignants, refusent une trop grande proximité avec les logiques répressives. Ils conservent avec l'institution judiciaire des relations distantes pour que leur mission première, l'action sociale ou thérapeutique, ne devienne pas un prétexte légitimant un surcroît de surveillance et de répression (De Maillard, 2001 ; Mouhanna, Ackermann, 2001 ; Gautron, 2009). Les tensions sont si nombreuses que l'on pourrait multiplier les exemples à l'envi. La police nationale ne voit pas toujours d'un très bon œil la concurrence des polices municipales (Le Goff, 2003). Les bailleurs sont désignés comme les principaux responsables de la dégradation des logements sociaux et corrélativement du climat dans les quartiers (Donzelot, Wyvekens, 2004). Quant aux professionnels de la sécurité privée, ils affrontent une suspicion quasi culturelle (Ocqueteau, 2004, 140).

Si l'on aborde ces divergences dans leur globalité, on remarque qu'elles s'alimentent de la crise d'identité qui traverse de nombreuses institutions. Les acteurs perçoivent la dynamique partenariale comme une intrusion de non-spécialistes dans leurs champs de compétence, et partant comme une contestation implicite de leurs savoirs professionnels (Ion, 1991 ; Roché, 1998). Au final, parquet contre corps préfectoral, magistrats contre policiers, éducateurs contre représentants de l'ordre et bénévoles, le milieu abonde de rivalités corporatistes qui attisent et rationalisent les attitudes de réserve, quand ce n'est pas d'hostilité, à l'égard des institutions voisines (Thoenig, 1994, 368). On se trouve donc confronté à un mythe partenarial qui occulte les relations conflictuelles entre acteurs (De Maillard, 2000b). La recherche de partenariat se heurte à des logiques de fermeture plus ou moins explicites: refus ouvert de collaborer, participation non effective (au mieux simple présence dans le collectif), retranchement derrière l'argumentaire du secret professionnel ou du "manque de temps», etc. Autant de stratégies ou de freins inconscients, qui dénotent une fois encore l'existence de "petits mondes", incapables de s'ouvrir parce qu'alimentés en permanence d'une force centripète et un souci de protection du pouvoir (Dhume, 2001, 144). Leur investissement dans les dispositifs partenariaux est donc le plus souvent limité.

\section{B - Une implication limitée dans les dispositifs de concertation}

Comme dans la plupart des États poursuivant une stratégie de coproduction de la sécurité (Crawford, 1998 ; Strebelle, 2002), ces multiples tensions ou oppositions ne se traduisent pas nécessairement par des conflits ouverts sur la scène locale (Le Goff, 2002b). Malgré le poids des logiques corporatistes et professionnelles, les acteurs évitent les situations de confrontation directe et privilégient une stratégie d'évitement. Sans refuser systématiquement 
les occasions de rencontre et de débat avec leurs éventuels partenaires, ils investissent peu les dispositifs partenariaux. Si les CLS ont permis de dynamiser le partenariat, les réunions interinstitutionnelles se présentent encore trop souvent comme de "grandes messes », au sein desquelles l'absentéisme est fréquent, les débats pauvres ou superficiels. Les personnels des conseils généraux, les magistrats du siège (juges des enfants, de l'application des peines), les services de l'administration pénitentiaire et de la PJJ comptent encore parmi les grands absents de la concertation (Duffe, Dupont, Steinmann, 2005). Les échanges interprofessionnels se raréfient fréquemment, les deux tiers des CLSPD ne se réunissant qu'une ou deux fois par an. Pour maintenir une unité de façade, les problèmes qui n'emportent pas consensus n'y sont pas évoqués, ou le sont en coulisses et en l'absence des voix les moins consensuelles sur le sujet (Le Goff, 2003, 349). Les acteurs opposent par ailleurs diverses résistances dans le processus de circulation des informations. De peur qu'elles soient utilisées pour discréditer leurs interventions, certains services refusent de divulguer leurs statistiques ou transmettent des données de qualité douteuse ${ }^{6}$. Les élus locaux dénoncent fréquemment la rétention d'informations de la part des autorités policières et judiciaires. En juillet 2005, une mission inter-inspections a certes noté de grands progrès dans l'information des élus (Duffe, Dupont, Steinmann, 2005). Les chiffres locaux de la délinquance leur sont généralement communiqués mensuellement et les informations transmises vont parfois au delà des obligations réglementaires et législatives. Récemment, le Conseil National des Villes a toutefois relevé que leur droit à l'information (art. L2211-3 du CGCT) n'était pas totalement respecté ${ }^{7}$. Des Directions départementales de sécurité publique rechignent à transmettre leurs informations sur l'état de la délinquance locale. Les élus déplorent surtout un manque d'informations de la part des services judiciaires, notamment sur le suivi des affaires signalées et sur les sortants de prison.

Les enseignants, les éducateurs de la PJJ, les travailleurs sociaux de l'aide sociale à l'enfance et les juges pour enfants résistent quant à eux à l'institutionnalisation des pratiques de « secret partagé ». Bien que consacrées par les lois du 5 mars 2007 relative à la prévention de la délinquance et à la protection de l'enfance (art. L121-6-2 et L226-2-2 du Code de l'action sociale et des familles), celles-ci sont loin de faire l'unanimité chez les professionnels du milieu médico-social. La plupart avancent des explications juridiques ou déontologiques pour s'opposer aux échanges d'informations nominatives (Marcus, 2003). Au delà de la question du secret professionnel, ils craignent de perdre le lien difficilement établi avec les jeunes, de passer à leurs yeux pour des délateurs (Donzelot, Wyvekens, 2004 ; Duval, Fontaine, 2000 ; Boucher, 2007). Si l'inscription législative du «secret partagé » a permis d'encadrer les échanges d'informations, il n'est pas certain qu'elle les favorise. Bien avant 2007, des acteurs ont accepté de communiquer sur des problématiques individuelles. L'absence de cadre juridique leur garantissait une totale liberté d'appréciation. Les échanges étant informels, réalisés au cas par cas et en fonction des relations de confiance établies avec leurs partenaires, ils ont eu le sentiment de sauvegarder leurs principes éthiques et se sont convaincus du bienfondé de leur démarche. Ils ont au contraire perçu la loi de 2007 relative à la prévention de la délinquance comme une atteinte à la règle du secret professionnel, comme l'expression d'un système de délation et de contrôle social (Boucher, 2007). Ces nouvelles dispositions législatives ont dès lors cristallisé les résistances des travailleurs sociaux, au point de

\footnotetext{
${ }^{6}$ Le diagnostic du premier CLS de l'agglomération nantaise illustre parfaitement ces pratiques. Alors que le rectorat disposait à l'époque d'un système trimestriel de collecte d'informations visant à répertorier les absentéismes et les actes de délinquance au sein des établissements scolaires, le rectorat signalait pour l'année 1997 un seul cas de consommation de stupéfiants et un seul cas de trafic dans deux collèges de l'agglomération. La véracité de ces chiffres semblait à ce point douteuse que le rédacteur du diagnostic, dont on ignore l'identité, avait accompagné la transcription de ces données de trois points d'exclamation.

${ }^{7} \mathrm{CNV}$, Avis sur la mise en $e u v r e$ de la loi prévention de la délinquance du 5 mars 2007, mars 2009.
} 
compromettre l'avenir des premières expériences de «secret partagé ». Depuis 2007, les dispositifs en place à Nantes, Saint-Herblain et Rezé se sont maintenus parce que les élus municipaux ont officieusement fait savoir aux travailleurs sociaux qu'ils n'appliqueraient pas la loi. Dans un courrier adressé aux maires du département, le président du Conseil général de Loire-Atlantique a quant à lui rappelé que les échanges d'informations devaient être réalisés avec le souci d'informer et d'associer les familles, et de limiter la communication aux strictes informations utiles à la résolution des difficultés dans un but clairement établi d'aide aux familles. Dans de nombreux sites, la loi de 2007 a toutefois renforcé les attitudes de retrait des travailleurs sociaux au sein des dispositifs de concertation ${ }^{8}$.

À ce stade de la réflexion, comment comprendre la variété des situations sur le terrain ? À partir de quelles variables expliquer la réussite du partenariat dans certains sites, et son échec ailleurs ? Si la structuration des réseaux d'action publique dépend incontestablement des positionnements politiques, institutionnels ou professionnels des différents acteurs, deux facteurs essentiels influent sur la dynamique partenariale: le volontarisme de quelques professionnels et la personnalisation des échanges. La concertation fonctionne lorsque les dispositifs locaux réunissent des acteurs qui, sans être des «marginaux » au sein de leurs services respectifs ${ }^{9}$, acceptent de sortir des schémas de pensée et d'action liés à leur appartenance institutionnelle ou professionnelle, des acteurs ouverts sur leur environnement, sans préjugés sur leurs partenaires et convaincus des bienfaits d'une approche transversale. De sorte que l'initiative ou la poursuite d'une démarche partenariale peut provenir selon les sites d'un préfet, d'un directeur départemental de la sécurité publique, d'un procureur, d'un maire, etc. Plusieurs études insistent également sur l'importance des relations personnalisées qui se nouent entre professionnels, indépendamment des rapports inter-institutionnels préexistants (Thoenig, 1994 ; Gaudin, 1999 ; Dubouchet, Berlioz, 2001 ; Tiévant, 2002). Lorsqu'ils établissent des liens privilégiés, éventuellement sur un registre affinitaire, les acteurs parviennent à désamorcer les situations conflictuelles pour s'inscrire dans une dynamique collective. Ces constats démontrent tout l'intérêt des enceintes de discussion installées à un niveau infra-municipal. Les instances de concertation rassemblent le plus souvent des responsables institutionnels. À la différence d'autres États, comme la Grande-Bretagne ou les États-Unis (Donzelot, Mevel, Wyvekens, 2003 ; Body-Gendrot, 1998), les tentatives pour favoriser la participation des agents « de première ligne » ont généralement échoué. Ceux-ci sont rarement consultés ou associés aux dispositifs de concertation, quand ils n'ignorent pas l'existence même des CLSPD et des CLS. Or, le fait que les responsables institutionnels entretiennent de bonnes relations n'induit pas de véritables rapprochements entre ceux qui, sur le terrain, sont quotidiennement appelés à coopérer (agents de police, travailleurs sociaux, enseignants, etc.). La transversalité hiérarchique n'a qu'un impact mineur sur le terrain. Les relations sont souvent tendues entre éducateurs et personnels de l'éducation nationale amenés à échanger à l'occasion du suivi de mineurs délinquants. Dans l'agglomération nantaise, les enseignants n'ont pas collaboré avec les éducateurs de la PJJ lorsque ces derniers sont intervenus dans les collèges pour mener des opérations de prévention «13-18 questions de justice ». Aux dires de la direction départementale de la PJJ, des éducateurs ont même reproché à des enseignants de ne pas y assister, alors même que l'opération se déroulait durant leurs cours et avec leurs élèves. De nombreux magistrats nantais portent un regard critique sur le fonctionnement de la PJJ et les pratiques des éducateurs. Ailleurs, des policiers investissent

\footnotetext{
${ }^{8}$ Ibid., 15 sqq.

${ }^{9}$ Dans un certain nombre de cas, les structures de concertation réunissent des exclus des institutions dominantes, qui trouvent là l'occasion d'une expression spécifique qui ne peut guère s'exercer habituellement dans la marche normale de l'institution (Ion, 1991, 72). Dans cette hypothèse, la concertation risque de tourner à vide, sans prise sur le cours de l'action publique.
} 
des établissements scolaires sans prévenir la direction, pour dépister la détention de stupéfiants ou pour interpeller sans un minimum de discrétion des mineurs suspectés d'infractions. Des travailleurs sociaux limitent les contacts avec la police nationale ou municipale, voire même avec des agents de médiation suspectés d'amateurisme. Les relations entre professionnels de terrain restent donc distantes, teintées selon les cas d'ignorance, d'incompréhension ou de méfiance. À l'inverse, les enceintes de discussion infra-municipales, comme les formations inter-institutionnelles, facilitent l'appropriation de la démarche partenariale par les personnels. Outre l'effet d'intercompréhension qu'elles génèrent, les agents ont le sentiment d'être écoutés et considérés par leurs supérieurs hiérarchiques, de participer véritablement à la conception et à la mise en œuvre des programmes. Parce qu'ils sont une source précieuse d'informations, les diagnostics et les évaluations gagnent en qualité. Ils assurent aux dispositifs une plus grande souplesse car les informations diffusées par la base permettent de réorienter l'action publique en continu.

Si l'investissement personnel de quelques professionnels garantit la qualité des échanges, il n'en fragilise pas moins les dispositifs de concertation. Puisque leur efficacité dépend d'implications individuelles, le départ des acteurs les plus mobilisés déstabilise inévitablement le réseau constitué, avec pour conséquence une perte des expériences acquises, voire une interruption de la démarche partenariale (Le Goff, 2003 ; Tiévant, 2002). La fréquence du turn over au sein de la fonction publique, notamment les mutations fréquentes du corps préfectoral et des magistrats, constitue dès lors un frein puissant au développement des coopérations (Donzelot, Wyvekens, 2004). À l'identique, la pérennité d'un projet peut être mise en péril par le renouvellement périodique des élus locaux. Ces investissements personnels présentent par ailleurs un risque d'essoufflement. Outre la complexité des rapports inter-institutionnels, il n'est pas rare que les «militants» de la coproduction subissent un phénomène de marginalisation au sein de leurs administrations d'origine. Leurs collègues et leurs hiérarchies restent souvent perplexes, voire hermétiques, face à de nouvelles méthodes d'action risquant de bouleverser leurs pratiques. Du fait de cette impossibilité à convaincre des vertus du projet partenarial, la démobilisation progressive des acteurs les plus engagés n'est pas improbable.

\section{C - Les résultats mitigés de la concertation}

La plupart des programmes locaux de lutte contre la délinquance demeurent mono-sectoriels (Mouhanna, 2005). Pour conserver leurs marges de manœuvre respectives, les acteurs multiplient les objectifs assignés aux contrats locaux de sécurité et retiennent des dénominations imprécises. Le premier CLS de l'agglomération nantaise présentait ainsi les objectifs suivants : lutter contre l'insécurité constatée, renforcer le sentiment de sécurité, promouvoir et développer la citoyenneté en améliorant la qualité de vie dans les quartiers, favoriser l'insertion des publics en difficulté. De tels relevés d'intentions permettent aux partenaires de s'entendre sur des mots communs, sans que ceux-ci aient la même signification pour chacun (De Maillard, 2000a). Le plan d'action étant réduit à une formalité protocolaire [...] sans autre signification concrète (Monjardet, 2002, 547), les acteurs se trouvent libérés de toute obligation au moment de la mise en œuvre des programmes, d'autant que leurs engagements sont généralement peu contraignants. Dans les CLS, peu de fiches-actions détaillent le contenu des activités, le calendrier de déroulement des opérations, le coût de mise en œuvre (Sina, 2002). Sous la forme de catalogues (Le Goff, 2003), ils rassemblent des actions que chaque institution conduit de façon autonome. Malgré les financements des collectivités locales de l'agglomération nantaise, l'institution judiciaire communique peu sur 
les opérations qui relèvent peu ou prou de la politique pénale (maisons de la justice et du droit, alternatives aux poursuites). Les élus reprochent au parquet son manque de transparence et leur exclusion du processus de définition de la politique pénale. Cette fermeture, que les magistrats justifient par leur indépendance, n'est pas le propre de l'institution judiciaire. La nature et le contenu des actions policières de prévention dans les établissements scolaires (de l'usage de stupéfiants, etc.) ne sont pas discutés préalablement avec le personnel enseignant. Comme indiqué précédemment, les interventions de la PJJ dans les collèges ne donnent lieu à aucune forme de coopération véritable. Les exemples abondent de ce manque d'investissement conjoint. "Assez rares sont, en définitive, les actions issues à proprement parler de la confrontation des constats, de la rencontre d'acteurs différents élaborant à partir de là des contenus communs. [...] la plupart des contrats ordonnent leurs fiches par institutions plutôt que par thèmes, ce qui reflète un modus operandi encore largement centré sur une prise d'initiative sectorielle (Donzelot, Wyvekens, 2004, 123).

L'atomisation de l'action publique locale s'explique également par la tentation qu'ont les acteurs d'instrumentaliser les dispositifs contractuels. Nombre de «partenaires » défendent leurs propres intérêts et tentent de maximiser leurs parts de crédit, d'où le manque de cohérence et la superposition des projets. Dans une lettre adressée au Premier ministre le 10 septembre 1984, G. Bonnemaison, alors vice-président du CNPD, déplorait déjà que les ministères s'efforcent de retrouver dans les dossiers pour lesquels ils se trouvent taxés, des actions relevant de leurs critères d'intervention habituels (cité in Chevalier, 1988, 239). Cette stratégie de captation des ressources ne s'est jamais démentie par la suite (Le Goff, 2003, 359). Plutôt que d'expérimenter des projets novateurs définis collectivement, certaines administrations ont utilisé des crédits contractualisés pour financer leurs opérations de fonctionnement courant: achat d'équipements individuels pour une brigade VTT (vélos, vêtements, matraques), vidéoprojecteur pour une brigade de prévention de la délinquance juvénile, achat de matériels informatiques ou audiovisuels pour des commissariats et des maisons d'arrêt, etc. (Cour des comptes, 2002). Des élus ont proposé des actions sans rapport avec les objectifs prioritaires des CLS Pour obtenir des crédits malgré le désaccord de leurs partenaires ou l'absence de lourds problèmes de délinquance dans leurs territoires, certains ont usé de leur influence politique, de leur présence aux échelons politiques supérieurs ou de leur accès direct aux administrations centrales (Mouhanna, 2005). Malgré l'opposition du procureur du TGI de Nantes, J. Floch, alors député et ancien maire de Rezé, est ainsi parvenu à obtenir l'accord du ministre de la Justice en 2001 pour l'installation d'une seconde MJD sur sa commune. Face à cette logique clientéliste et pour ne pas attiser les contestations, les préfets choisissent fréquemment de répartir les crédits entre toutes les communes, quelle que soit la gravité des difficultés rencontrées (Body-Gendrot, Duprez, 2001). Cette stratégie explique le caractère inflationniste de la géographie prioritaire, et partant la pratique de saupoudrage des crédits. Selon la première mission interministérielle d'évaluation des CLS, près de la moitié des contrats ont été signés en dehors des départements les plus sensibles (Dieu, Domingo, 2003). Les subventions ministérielles ne sont donc pas concentrées sur les territoires les plus affectés par l'insécurité, alors même qu'ils se situent généralement dans les communes disposant des plus faibles moyens. Cette situation introduit des inégalités entre collectivités, les communes pauvres devant assumer des charges plus lourdes en matière de prévention et de sécurité. Les subventions de l'État ne sont donc pas toujours utilisées à bon escient, ou du moins pour la mise en œuvre d'opérations contribuant directement à la prévention ou à la répression de la délinquance. Pour éviter de telles dérives, les circulaires relatives au FIPD précisent que les subventions perçues ne doivent pas se substituer aux crédits de droit commun, notamment pour l'achat d'équipements, et qu'elles n'en sont que le complément. Les préfets sont par ailleurs invités à rejeter les actions imprécises dans leur objet ou leur libellé. Aux fins de vérification, $20 \%$ des organismes subventionnés devront être 
contrôlés par les services de l'État et les sommes restituées en cas d'utilisation non-conforme aux objectifs fixés. Reste à savoir si ces injonctions seront suivies d'effet, les moyens de contrôle des préfectures étant plus théoriques qu' effectifs (Le Goff, 2002a ; Gautron, 2006).

\section{Conclusion}

$\mathrm{Au}$ terme de cette analyse, l'appréciation portée sur les résultats de la coproduction de la sécurité ne peut être que nuancée. Malgré quelques exemples de partenariats réussis, qui démontrent que les coopérations sont possibles et bénéfiques, il existe un écart important entre la philosophie affichée par la doctrine de la coproduction et les pratiques de concertation. Nous sommes encore bien loin de la cohérence, de la cohésion, de la complémentarité et du consensus, ces qualités tant attendues d'une approche partenariale très certainement idéalisée. Hostiles à l'émergence d'obligations réciproques, la plupart des professionnels acceptent de dialoguer, de s'associer ponctuellement, mais non de collaborer durablement pour la mise en œuvre de projets conjoints. D'où les maigres résultats de la loi du 5 mars 2007 relative à la prévention de la délinquance, dont l'un des objectifs était la relance et l'approfondissement du partenariat. Le nombre de CLS (687 en 2006, 414 début 2009) et de CLSPD (867 en 2006, 790 début 2009) diminue. Peu de contrats locaux de sécurité « nouvelle génération » ont été signés (22 en 2007, 6 en 2008) ${ }^{10}$ et les dispositifs partenariaux sont en sommeil dans de nombreux sites. La coopération intercommunale s'est ainsi considérablement restreinte dans l'agglomération nantaise. Si des discussions sont engagées concernant la signature d'un CLS «nouvelle génération», celui-ci ne serait plus intercommunal, la municipalité de Nantes privilégiant de nouveau des relations bilatérales avec la préfecture et ses autres partenaires. Au-delà, ce prochain contrat institutionnaliserait une contractualisation sans coproduction, les négociations en cours manifestant un net repli des acteurs locaux sur leurs compétences respectives.

Lorsque l'on quitte le terrain du système d'action pour observer ce qu'il produit, c'est-à-dire la substance des politiques de lutte contre la délinquance, les changements paradigmatiques majeurs qui affectent ce champ d'action depuis 2002 nourrissent quelques craintes quant à l'avenir de l'approche partenariale. Dans le champ pénal, les exigences du nouveau droit à la sécurité supplantent progressivement celles du droit à la sûreté, au point de consacrer un modèle de politique criminelle «libéral-autoritaire» (Foessel, 2005; Gautron, 2006) ou « garantiste-sécuritaire » (Danet, 2008). Pour dissuader et réprimer les comportements d'écart aux normes stimulant le sentiment d'insécurité, les incriminations et les sanctions pénales se multiplient. Pour systématiser la réaction pénale, les agents de répression disposent de prérogatives élargies, les dispositifs de surveillance se généralisent et les procédures pénales s'accélèrent. Absorbée par le concept de «sécurité », la prévention se trouve de plus en plus circonscrite à la dissuasion pénale et à la sécurité des victimes potentielles, à la surveillance, au contrôle et à la neutralisation des délinquants. Les pouvoirs publics n'ont pas abandonné les politiques de prévention sociale qui ont singularisé le modèle français au plan international (Gautron, 2006). Toutefois, dans un contexte de négation des paramètres psychosociaux pour expliquer le passage à l'acte, leurs stratégies se modifient subrepticement. Les programmes de prévention sociale introduits ces dernières années présentent systématiquement une dimension morale de responsabilisation, de dépistage, de contrôle, d'encadrement ou de rappel à l'ordre (contrat de responsabilité parentale, conseil des droits et devoirs des familles notamment). Sans que cet objectif soit formellement énoncé, l'efficacité préventive se trouve recherchée au

\footnotetext{
${ }^{10}$ Source: statistiques du ministère de l'Intérieur et du Secrétariat général du Comité interministériel de prévention de la délinquance.
} 
travers de la restauration de l'autorité des familles, des institutions, de la loi. Nous sommes dès lors bien loin de la politique sociale d'émancipation inaugurée il y a plus de vingt ans. Au-delà, la prévention sociale se trouve concurrencée par de nouvelles modalités préventives dont les fondements et les outils diffèrent totalement. Les interventions correctives sur le milieu social et familial cèdent partiellement la place à des opérations de prévention situationnelle chargées de restaurer la sécurité immédiate de la population et/ou l'efficacité policière (Gautron, 2006).

Ces mouvements contemporains de politique criminelle opèrent pourtant au détriment de la dynamique partenariale. Les appels à un durcissement de la répression confortent les agents des administrations régaliennes dans l'idée qu'ils sont les seuls responsables, sinon les seuls compétents, pour enrayer l'insécurité. La concertation serait utile aux autres, appelés à comprendre et relayer leurs actions, mais pas pour eux-mêmes, hormis d'un point de vue informationnel. La pression sécuritaire tend par ailleurs à transformer les services de police et les magistrats en concurrents, voire en opposants. Puisque le sens commun soutient que la réduction de la délinquance dépend uniquement d'une répression efficace, la montée des violences est perçue comme la conséquence de défaillances des maillons de la chaîne pénale. Lorsque les résultats obtenus ne sont pas à la hauteur des promesses gouvernementales, justice et police se renvoient dos à dos la responsabilité de cet échec. Parmi les membres du système d'action, certains n'opposeront pas de véritables résistances à cette tentative de leadership, du moment qu'ils puissent se décharger des illégalismes qui les affectent sur les autorités régaliennes et ainsi éviter d'aborder de front la question de leur éventuelle participation à la multiplication des tensions. À l'inverse, les réticences des travailleurs sociaux et des soignants risquent de se cristalliser face à des dispositifs partenariaux qu'ils appréhendent comme des outils de contrôle social indifférents aux problématiques psychosociales, à leur éthique et à leurs pratiques professionnelles. La peur d'être instrumentalisés par les institutions régaliennes pour servir, grâce aux informations qu'ils détiennent, l'alimentation de la chaîne pénale, exacerbe leurs positions de retrait. À terme, il n'est pas improbable que la concertation se résume aux questions de répression et de sécurité, à l'exclusion de toute réflexion sur la prévention sociale. En toute hypothèse, les rivalités institutionnelles, les positions défensives et les implications a minima dans les dispositifs partenariaux ont de beaux jours devant elles. 


\section{Bibliographie}

ARNAUd A.-J., 1999, La régulation par le droit en contexte globalisé, in COMMAILlE J., JoBERT B. (dir.), Les métamorphoses de la régulation politique, Paris, LGDJ, Maison des Sciences de l'Homme, 147-175.

BARON C., 2003, La gouvernance : débats autour d'un concept polysémique, Droit et Société, 54, 329-349.

Body-GENDrot S., 1998, Les villes face à l'insécurité. Des ghettos américains aux banlieues françaises, Paris, Bayard.

Body-Gendrot S., DupreZ D., 2001, Les politiques de sécurité et de prévention dans les années 1990 en France, Déviance et Société, 25, 4, 377-402.

BouCHER M., 2007, Les travailleurs sociaux face aux bandes. Stigmatisation de la jeunesse populaire et instrumentalisation des acteurs sociaux, in MoHAMmed M., MuCCHIELli L. (dir.), Les bandes de jeunes. Des «blousons noirs » à nos jours, Paris, La Découverte, 217-244.

Chevalier G., 1988, L'intérêt central pour le local. Analyse des politiques socio-préventives en France entre 1981 et 1986, Déviance et Société, 12, 3, 237-267.

CHevalier G., 1990, Administrations de mission et luttes d'influence. Le cas du secteur socio-préventif, Revue française de sociologie, 31, 421-437.

Chevallier J., Morand C.-A., 1991, L'État propulsif. Contribution à l'étude des instruments d'action de l'État, Paris, Publisud.

COMMISSION DES MAIRES SUR LA SECURITE, 1982, Face à la délinquance : prévention, répression, solidarité, Rapport au Premier ministre, Paris, La documentation française.

COUR DES COMPTES, 2002, La politique de la ville, Rapport au Président de la République

CRAWFORD A., 1998, Partenariat et responsabilité à l'ère managériale. Retour sur l'expérience britannique, Les Cahiers de la sécurité intérieure, 33, 51-87.

CRAWFORD A., 2001a, Les politiques de sécurité locale et de prévention de la délinquance en Angleterre et au Pays de Galles : nouvelles stratégies et nouveaux développements, Déviance et Société, 25, 4, 427-458.

CRAWFORD A., 2001b, Vers une reconfiguration des pouvoirs ? Le niveau local et les perspectives de la gouvernance, Déviance et Société, 25, 1, 3-32.

CROZIER M., 1997, État modeste, État moderne. Stratégies pour un autre changement, 3 éd., Paris, Fayard.

DAMAMme D., JoberT B., 1995, La politique de la ville ou l'injonction contradictoire en politique, Revue Française de science politique, 45, 1, 3-30.

DANET J., Cinq ans de frénésie pénale, in Mucchielli L., La frénésie sécuritaire. Retour à l'ordre et nouveau contrôle social, Paris, La découverte, 19-29.

DE MAIllard J., 2000a, La politique de la ville: une institutionnalisation inachevée, Thèse, Bordeaux, Université Montesquieu - Bordeaux IV.

DE MAILlARD J., 2000b, Le partenariat en représentations : contribution à l'analyse des nouvelles politiques sociales territorialisées, Politiques et management public, 18, 3, 21-41.

De Maillard J., 2001, Les travailleurs sociaux en interaction. Politiques sociales urbaines, mobilisation des professionnels et fragmentations, Sociologie du travail, 44, 215-232.

De Maillard J., 2003, Vers des politiques locales de sécurité, in Roche S. (dir.), En quête de sécurité. Causes de la délinquance et nouvelles réponses, Paris, A. Colin, 283-295.

DE MAILlard J., 2008, Le magistrat, le maire et la sécurité publique: action publique partenariale et dynamiques professionnelles, Revue française de sociologie, 4, 793-818.

Dieu F., Domingo B., 2003, Partenariat et évaluation: le cas des contrats locaux de sécurité, Revue Internationale de Criminologie et de Police Technique, LVI, 2, 3-22.

DHUME F., 2001, Du travail social au travail ensemble. Le partenariat dans le champ des politiques sociales, Paris, éd. A.S.H.

DONZELOT J., ESTEBE P., 1994, L’ État animateur. Essai sur la politique de la ville, Paris, éd. Esprit. 
Donzelot J., Mevel C., WyveKens A., 2003, Faire société. La politique de la ville aux États-Unis et en France, Paris, Seuil.

DonZelot J., WyVEKENS A., 2004, La magistrature sociale. Enquêtes sur les politiques locales de sécurité, Paris, La documentation française.

Dubouchet L., Berlioz G., 2001, L'intervention de l'institution judiciaire dans les contrats locaux de sécurité, Rapport d'évaluation, ministère de la Justice, non publié.

DufFe P., DuPONT M., STEINMANN B. et al., 2005, Propositions d'orientations pour la mise en place de contrats locaux de sécurité de nouvelle génération, Paris, Inspection générale de l'administration.

Dupont B., 2008, La gouvernance et la sécurité, in Cusson M., DupOnT B., LeMIEuX F. (dir.), Traité de sécurité intérieure, Lausanne, Presses polytechniques et universitaires romandes, 67-80.

Duval M., FonTAINE A., 2000, Quand des pratiques différentes se heurtent : les relations entre les travailleurs de rue et les autres intervenants, Nouvelles pratiques sociales, 13, 1, 49-67.

EPSTEIN R., 2008, Gouverner à distance : la rénovation urbaine, démolition-reconstruction de l'appareil d'État, Thèse, Cachan, ENS.

ESTEBE P., 1999, L’usage des quartiers. Action publique et géographie dans la politique de la ville (1989-1998), Thèse, Paris, Université de Paris X - Nanterre.

FoESSEL M., 2005, Légitimation de l'État. De l'affaiblissement de l'autorité à la restauration de la puissance, Esprit, 3-4, 242-256

Froment J.-C., 1999, Le maire et la sécurité, Revue Française d'Administration Publique, 91, 455-469.

GAUdIN J.-P., 1999, Gouverner par contrat. L’action publique en question, Paris, Presses de sciences po.

GAUDIN J.-P., 2001, Le sens du contrat dans les politiques publiques, Esprit, 2, 112-121.

GaUtron V., 2006, Les politiques publiques de lutte contre la délinquance, Thèse, Nantes, Université de Nantes.

GaUtron V., 2007, La fin de la singularité du modèle français de prévention de la délinquance, Actualité Juridique Pénale, 5, 205-209.

Gautron V., 2008, L'évaluation de la politique criminelle : des avancées en trompe-1'œil ?, Archives de politique criminelle, 30, 201-219.

GAUTRON V., 2009, De la société de surveillance à la rétention de sûreté : étapes, faux-semblants, impasses et fuite en avant, Actualité Juridique Pénale, 2, 54-57.

GLEIZAL J.-J., 1995, Le préfet dans le champ de la sécurité et de la prévention de la délinquance, in GLEIZAL J.-J. (dir.), Le retour des préfets ?, Grenoble, Presses Universitaires de Grenoble, 115-128.

GLEIZAL J.-J., 2003, L'élaboration des politiques de sécurité en France (1982-2002), in FrOMENT J.-C., GLEIZAL J.-J., KAluSZYNSKi M. (dir.), Les États à l'épreuve de la sécurité, Grenoble, Presses Universitaires de Grenoble, 31-45.

GorgeOn C., Estebe P., LEON H., 2000, De la prévention sociale à la tranquillité publique. Glissement sémantique et renouveau de l'action publique, Les Cahiers de la sécurité intérieure, 39, 223-241.

HASSID O., 2005, La gouvernance de sécurité : un concept pour un nouveau paradigme en criminologie ?, Revue Internationale de Criminologie et de Police Technique, LVIII, 2, 151-161.

ION J., 1991, Le travail social à l'épreuve du territoire, Paris, Privat.

LE GAles P., 1998, Régulation, gouvernance et territoire, in COMMAILle J., Jobert B. (dir.), Les métamorphoses de la régulation politique, Paris, LGDJ, Maison des Sciences de l'Homme, 203-240.

LE GOFF T., 2002a, Intercommunalité et sécurité. Une approche comparative de trois agglomérations, Paris, IHÉSI, coll. Études et Recherches.

LE GofF T., 2002b, Les contrats locaux de sécurité à l'épreuve du terrain. Réflexions sur l'action publique locale en matière de sécurité, Politiques et management public, 20, 1, 105-119.

LE GOFF T., 2003, Le maire garant de la tranquillité publique. Réactivation d'un rôle politique et recomposition de l'action publique en matière de sécurité quotidienne, Thèse, Rennes, Université de Rennes I. 
LE GofF T., 2004, Réformer la sécurité par la coproduction : action ou rhétorique ?, in RocHE S. (dir.), Réformer la police et la sécurité, Paris, O. Jacob, 81-104.

MARCOU G., 1997, La coopération contractuelle, la ville et le droit, in MARCOU G., RANGEON F., THIEBAULT J.L. (dir.), La coopération contractuelle et le gouvernement des villes, Paris, L'Harmattan, 47-120

MARCUS M., 2003, Veille éducative, secret professionnel et partage des informations, in COLLECTIF, Veille éducative. Une démarche collégiale de prévention des ruptures scolaires et éducatives, $1^{\mathrm{e}}$ rencontre nationale, Paris, DIV, 16-20.

MONJARDET D., 2002, L’insécurité politique : police et sécurité dans l'arène électorale, Sociologie du travail, 44, 543-555.

MoRAND C.-A., 1999, Le droit néo-moderne des politiques publiques, Paris, L.G.D.J, Maison des Sciences de l'homme, coll. Droit et Société, vol. 26.

Mothes F., Novaro P., PAsinetTi P., 2005, Besoins et modalités d'échanges entre acteurs publics et privés de la sécurité, Les Cahiers de la sécurité, 57, 253-274.

MouhanNA C., 2005, Coproduction, cohérence ou concurrence ? Réflexions sur la coopération élus/policiers en matière de sécurité, in FERret J., Mouhanna C. (dir.), Peurs sur les villes. Vers un populisme punitif à la française, Paris, PUF, 103-120.

MOUHANnA C., ACKERMANN W., 2001, Le parquet en interaction avec son environnement : à la recherche des politiques pénales, Rapport de recherche, Mission de recherche Droit et Justice, Paris, ministère de la justice.

Ocqueteau F., 2004, Polices entre Etat et marché, Paris, Presses de Sciences Po.

OcQueteau F., FRenais J., VARly P., 2002, Ordonner le désordre. Une contribution au débat sur les indicateurs du crime, Paris, IHÉSI,/La documentation française.

Ost F., VAn De Kerchove M., 2002, De la pyramide au réseau? Pour une théorie dialectique du droit, Bruxelles, Publications des Facultés universitaires Saint-Louis.

PAPADOPOUlos Y., 1995, Complexité sociale et politiques publiques, Paris, Montchrestien.

PAPAdOPOUlos Y., 2001, Transformations du style de l'action publique et responsabilité politique, Politiques et management public, 19, 1, 165-184.

ROBERT Ph., 1999, Le citoyen, le crime et l'État, Genève, Librairie Droz.

Roche S., 1998, Sociologie politique de l'insécurité, Paris, PUF.

RochE S., 2004, Vers la démonopolisation des fonctions régaliennes : contractualisation, territorialisation et européanisation de la sécurité intérieure, Revue Française de Science Politique, 54, 1, 43-70.

SinA F., 2002, Contrats locaux de sécurité. La montée en puissance de la prévention situationnelle, Les Cahiers de la sécurité intérieure, 50, 73-99.

STREBElle C., 2002, Les contrats de sécurité. Évaluation des politiques de prévention en Belgique, Bruxelles, Bruylant.

TIEvant S., 2002, Partenariat et police de proximité. Dilution ou consolidation des spécificités professionnelles ?, Les Cahiers de la sécurité intérieure, n48, 149-170.

Thoenig J.-C., 1994, La gestion systémique de la sécurité publique, Revue Française de Sociologie, 15, 357392.

WyVeKens A., 1997, L’insertion locale de la justice pénale. Aux origines de la justice de proximité, Paris, L'Harmattan. 